R. K. Simmons $\cdot$ A.-H. Harding $\cdot$ R. W. Jakes $\cdot$

A. Welch · N. J. Wareham · S. J. Griffin

\title{
How much might achievement of diabetes prevention behaviour goals reduce the incidence of diabetes if implemented at the population level?
}

Received: 7 September 2005 / Accepted: 28 November 2005 / Published online: 1 March 2006

C) Springer-Verlag 2006

\begin{abstract}
Aims/hypothesis: Randomised trials targeting high-risk people with impaired glucose tolerance have halved progression to diabetes using behavioural interventions aimed at achieving five goals related to weight, diet and physical activity. The number of people currently meeting these goals in the general population is unknown. The potential impact on the incidence of diabetes of increasing the proportion of people who meet these goals is also unclear. We quantified the association between the achievement of behavioural goals for the prevention of diabetes and the incidence of diabetes in a population-based cohort study. Subjects and methods: European Prospective Investigation into Cancer (EPIC)-Norfolk is a prospective cohort of 24,155 participants aged 40-79 years who attended a baseline health check and completed validated diet and activity questionnaires. We assessed the association between achievement of five diabetes healthy behaviour prevention goals (BMI $<25 \mathrm{~kg}$ / $\mathrm{m}^{2}$, fat intake $<30 \%$ of energy intake, saturated fat intake $<10 \%$ of energy intake, fibre intake $\geq 15 \mathrm{~g} / 4,184 \mathrm{~kJ}$, physical activity $>4 \mathrm{~h} /$ week) and risk of developing diabetes at follow-up (mean 4.6 years). Results: Only $20 \%$ of EPIC participants met three or more diabetes prevention goals. Diabetes incidence was inversely related to the number of goals achieved $(p<0.001)$. None of the participants who met all five goals developed diabetes, whereas diabetes incidence was highest in those who did not meet any goals. If the entire population were able
\end{abstract}

R. K. Simmons · A.-H. Harding $\cdot$ R. W. Jakes $\cdot$ N. J. Wareham S. J. Griffin $(\bowtie)$

MRC Epidemiology Unit, Strangeways Research Laboratory, Wort's Causeway,

Cambridge CB1 8RN, UK

e-mail: simon.griffin@mrc-epid.cam.ac.uk

Tel.: +44-1223-740044

Fax: +44-1223-740050

A. Welch

Department of Public Health and Primary Care,

University of Cambridge, Strangeways Research Laboratory,

Cambridge, UK to meet one more goal, the total incidence of diabetes would be predicted to fall by $20 \%$. Conclusions/ interpretation: In this population-based study, the risk of diabetes was inversely associated with the number of behaviour goals for diabetes prevention that were met. Interventions that promote achievement of these goals in the general population could significantly reduce the growing burden of diabetes-related morbidity and mortality.

Keywords Behaviour - Diabetes - Incidence · Intervention $\cdot$ Prevention

Abbreviations DPS: Diabetes Prevention Study - EPIC: European Prospective Investigation into Cancer

\section{Introduction}

Intensive behavioural interventions aimed at high-risk people have halved progression to diabetes in three different randomised trials [1-3]. In the large US Diabetes Prevention Program the risk reduction in the lifestyle arm was $58 \%$. An identical risk reduction was seen in the Finnish Diabetes Prevention Study (DPS), in which the intervention was aimed at increasing adherence to five lifestyle behaviour targets including nutritional intake, physical activity and weight reduction. These trials have clearly demonstrated reduced progression to diabetes in a high-risk group defined by having impaired glucose tolerance, but whether the impact would be similar in larger groups of individuals at lower risk is unknown. This cannot be directly measured at present in the absence of a clinical trial of behaviour change in unselected individuals. However, it may be estimated from observational data in a cohort study.

If the behaviours targeted by intervention trials have an impact at the population level, this information could be used to develop interventions applicable to larger groups of people at lower absolute risk. We assessed whether meeting five healthy goals similar to those described in the Finnish DPS (BMI $<25 \mathrm{~kg} / \mathrm{m}^{2}$, fat intake $<30 \%$ of energy intake, 
saturated fat intake $<10 \%$ of energy intake, fibre intake $\geq 15 \mathrm{~g} / 4,184 \mathrm{~kJ}$, physical activity $>4 \mathrm{~h} /$ week) was associated with reduced risk of developing diabetes in a population-based cohort study.

\section{Subjects and methods}

The European Prospective Investigation into Cancer (EPIC)-Norfolk is a prospective cohort study which recruited men and women aged 40-74 from general practices in the Norfolk region of the UK. Full details of the population are reported elsewhere [4]. In brief, between 1993 and 1998, 77,630 individuals were invited to take part in the study. Of these, 25,633 (33\%) consented and attended a baseline health check. This included anthropometric and blood pressure measurements and completion of validated diet and physical activity questionnaires $[5,6]$. The dietary questionnaire was a semiquantitative, selfadministered Food Frequency Questionnaire (FFQ) that was designed to measure an individual's habitual food and nutrient intake during the past year. This questionnaire has been validated for nitrogen, potassium, vitamin $\mathrm{C}$, sodium and energy intakes $[5,7]$. A health and lifestyle questionnaire included questions on occupational and nonoccupational physical activity [6]. Data were also collected on education levels, socio-economic background, family history of diabetes and smoking and alcohol consumption. In terms of anthropometric variables, blood pressure and serum lipids, EPIC-Norfolk participants were similar to a nationally representative sample [4], although the Norfolk region is slightly healthier than the general UK population, with a standardised mortality ratio of 94 [8]. There was a second health check between 1998 and 2000, and 15,028 (58.6\%) participants returned for this assessment.

We specified five diabetes prevention behaviour goals similar to those described in the DPS, to assess which behaviours predicted the risk of progression to diabetes. We simplified the first DPS goal, concerning loss of more than $5 \%$ body weight, to look at whether participants had a BMI of more or less than $25 \mathrm{~kg} / \mathrm{m}^{2}$ at baseline. The EPIC cohort does have records for weight at follow-up, but as all other exposure variables concerned data collected at baseline and the incidence of diabetes was assessed from baseline, it was decided to use baseline BMI for consistency. However, in order to avoid underestimating the benefits of weight reduction in seriously obese individuals by dichotomising the weight target, we also explored weight difference in the baseline data. We calculated the relative risk reduction for each $1.5 \mathrm{~kg} / \mathrm{m}^{2}$ difference in BMI, which is roughly equivalent to a $5 \%$ reduction in weight for most people. In the DPS, the frequency of physical activity was assessed using a fourpoint scale-those who reported 'walking, cycling or exercising lightly for at least $4 \mathrm{~h}$ per week' were said to have achieved a healthy goal. We created a similar composite measure of physical activity which summed the number of hours each participant reported spending on cycling, walking and 'other' exercise activities per week.
Participants who reported more than $4 \mathrm{~h}$ of these activities were likewise said to have met a healthy goal.

The three remaining nutritional goals (fat intake $<30 \%$ of energy intake, saturated fat intake $<10 \%$ of energy intake and fibre intake $\geq 15 \mathrm{~g} / 4,184 \mathrm{~kJ}$ ) were calculated directly for the EPIC population. Respondents were asked to estimate how frequently they had eaten items on a list of 130 foods over the past year, by choosing one of nine categories of response, ranging from never to more than six times a day. Portion weights and representative food codes were assigned to each item, and the nutritional value was calculated using the Compositional Analyses from Frequency Estimates (CAFE) program - a computer program that was developed alongside software for data entry of EPIC dietary information [9]. Daily intakes of fat, saturated fat, fibre and energy were calculated from the FFQ to assess possible achievement of the diabetes behaviour goals.

By summing the number of diabetes prevention behaviour goals that each participant met at baseline, a summary score was calculated with a range of six possible values $(0-5)$. Individuals with self-reported diabetes at baseline were excluded $(n=583)$ and those with missing data for healthy goal exposure variables $(n=902)$ were excluded, leaving 24,155 people for analysis. Incident cases of diabetes were ascertained from multiple sources including follow-up health checks, hospital and general practice registers and prescribing data, as well as an $\mathrm{HbA}_{1 \mathrm{c}}$ level of greater than $7 \%$ at either baseline or follow-up. Research has shown that a value of $\mathrm{HbA}_{1 \mathrm{c}}$ of $7 \%$ has the maximum specificity and sensitivity for detecting diabetes [10]; $\mathrm{HbA}_{1 \mathrm{c}}$ has also been shown to predict nephropathy and retinopathy as well as fasting blood glucose and 2-h postchallenge blood glucose [11]. Capture-recapture analysis indicated that $99 \%$ case ascertainment was attained [12].

\section{Statistical analysis}

Baseline characteristics were compared across the five diabetes prevention behaviour goals using one-way ANOVA for continuous variables and the $\chi^{2}$ test for trend for categorical variables. Social class was recoded as a binary variable based on the UK Registrar General's occupational classification (I-IIIa vs IIIb-V), and education was reclassified according to age on leaving full-time education ( $\leq 16$ vs $>16$ years). Years of follow-up was estimated as time from baseline to the first report of diabetes from any of the sources listed above. For each diabetes prevention score $(0-5)$, incidence rates per 1,000 person-years of follow-up were calculated. We also conducted a Cox regression analysis to investigate the crude and adjusted hazard ratios for diabetes incidence for each diabetes prevention score. We examined the rate of diabetes incidence across each of the diabetes prevention behaviours after recoding them into quintiles. The association between diabetes prevention behaviour scores and incident diabetes was assessed using multivariate logistic regression. We controlled for age, sex, social class, family 
history of diabetes, and prescription of antihypertensive medication. We undertook a sensitivity analysis for missing healthy goal exposure variables by recoding them to maximum or minimum values to assess the impact of missing data on our model. We also stratified our model by sex to see if there were any differences between the proportions of males and females meeting the healthy behaviour goals. All analyses were completed using Stata, version 8.0. (STATA, College Station, TX, USA).

The EPIC-Norfolk study was approved by the Norfolk Local Research Ethics Committee and participants gave written consent prior to the first health check.

Table 1 Baseline characteristics according to the number of diabetes healthy behaviour goals achieved: the EPIC-Norfolk study 1993-2000 $(n=24,155)$

\begin{tabular}{|c|c|c|c|c|c|c|}
\hline \multirow[t]{2}{*}{ Characteristic $^{\mathrm{a}}$} & \multicolumn{6}{|c|}{ Diabetes healthy behaviour goals } \\
\hline & 0 & 1 & 2 & 3 & 4 & 5 \\
\hline All respondents $(\%)$ & $2,516(10.4)$ & $9,540(39.5)$ & $7,473(30.9)$ & $3,009(12.5)$ & $1,414(5.9)$ & $203(0.8)$ \\
\hline \multicolumn{7}{|l|}{$\operatorname{Sex}^{b}$} \\
\hline Male $(\%)$ & $1,312(12.1)$ & $4,815(44.4)$ & $3,045(28.1)$ & $1,208(11.2)$ & $418(3.9)$ & $36(0.3)$ \\
\hline Female $(\%)$ & $1,204(9.0)$ & $4,725(35.5)$ & $4,428(33.2)$ & $996(13.5)$ & $996(7.5)$ & $167(1.3)$ \\
\hline Mean age, years $(\mathrm{SD})^{\mathrm{c}}$ & $60.4(9.5)$ & $59.5(9.2)$ & $58.5(9.4)$ & $59.0(8.9)$ & $57.9(9.1)$ & $57.9(8.5)$ \\
\hline \multicolumn{7}{|l|}{ Social class $(\%)^{\mathrm{b}}$} \\
\hline I-IIIa & $1,333(9.3)$ & $5,398(37.7)$ & $4,584(32.1)$ & $1,906(13.3)$ & $950(6.6)$ & $133(0.9)$ \\
\hline IIIb-V & $1,099(11.7)$ & $3,944(42.4)$ & $2,783(29.5)$ & $1,049(11.1)$ & $435(4.6)$ & $69(0.7)$ \\
\hline \multicolumn{7}{|l|}{ Age left full-time education $(\%)^{\mathrm{b}}$} \\
\hline$\leq 16$ years & $1,455(11.5)$ & $5,013(39.8)$ & $3,813(30.2)$ & $1,527(12.1)$ & $696(5.5)$ & $108(0.9)$ \\
\hline$>16$ years & $1,061(9.2)$ & $4,527(39.2)$ & $3,660(31.7)$ & $1,482(12.9)$ & $718(6.2)$ & $95(0.8)$ \\
\hline \multicolumn{7}{|l|}{ Family history of diabetes (\%) } \\
\hline Yes & $354(11.7)$ & $1,191(39.4)$ & $882(29.2)$ & $402(13.3)$ & $169(5.6)$ & $22(0.7)$ \\
\hline No & $2,162(10.2)$ & $8,347(39.5)$ & $6,588(31.2)$ & $2,606(12.3)$ & $1,245(5.9)$ & $181(0.9)$ \\
\hline \multicolumn{7}{|l|}{ Smoking status $(\%)^{\mathrm{d}}$} \\
\hline Current & $345(12.3)$ & $1142(40.8)$ & $948(33.9)$ & $260(9.3)$ & $96(3.4)$ & $9(0.3)$ \\
\hline Ex-smoker & $1,101(11.0)$ & $4,073(40.6)$ & $2,886(28.8)$ & $1,344(13.4)$ & $558(5.6)$ & $71(0.71)$ \\
\hline Never & $1,045(9.4)$ & $4,247(38.1)$ & $3,583(32.2)$ & $1,388(12.5)$ & $751(6.7)$ & $123(1.1)$ \\
\hline Mean daily alcohol consumption, g/day $(\mathrm{SD})^{\mathrm{e}}$ & $7.0(10.8)$ & $8.1(11.8)$ & $8.5(12.5)$ & $12.0(17.3)$ & $9.8(14.2)$ & $5.3(8.2)$ \\
\hline \multicolumn{7}{|l|}{ Mean waist circumference, $\mathrm{cm}(\mathrm{SD})^{\mathrm{c}}$} \\
\hline Male & $100.8(8.6)$ & $97.0(8.8)$ & $90.9(9.1)$ & $94.6(9.3)$ & 89.5 (7.9) & $85.6(8.0)$ \\
\hline Female & $89.7(10.5)$ & $85.3(10.5)$ & $77.9(9.3)$ & $81.2(9.8)$ & $77.0(8.6)$ & $73.8(5.4)$ \\
\hline \multicolumn{7}{|l|}{ Mean hip circumference, $\mathrm{cm}(\mathrm{SD})^{\mathrm{c}}$} \\
\hline Male & $105.5(5.8)$ & $104.1(5.9)$ & $100.1(5.9)$ & $102.1(6.3)$ & $99.2(5.7)$ & $96.2(4.8)$ \\
\hline Female & $109.3(9.1)$ & $106.0(9.0)$ & $100.1(8.0)$ & $103.0(8.7)$ & $99.6(7.3)$ & $97.0(4.7)$ \\
\hline \multicolumn{7}{|l|}{ Serum lipids, mmol/1 (SD) } \\
\hline Mean total cholesterol ${ }^{\mathrm{c}}$ & $6.3(1.2)$ & $6.3(1.2)$ & $6.1(1.2)$ & $6.2(1.1)$ & $6.0(1.3)$ & $6.0(1.1)$ \\
\hline Mean $\mathrm{LDL}^{\mathrm{c}}$ & $4.0(1.0)$ & $4.1(1.0)$ & $3.9(1.0)$ & $3.9(1.0)$ & $3.8(1.1)$ & $3.9(1.0)$ \\
\hline Mean $\mathrm{HDL}^{\mathrm{c}}$ & $1.3(0.4)$ & $1.4(0.4)$ & $1.5(0.4)$ & $1.4(0.4)$ & $1.5(0.4)$ & $1.6(0.4)$ \\
\hline Mean triglycerides ${ }^{\mathrm{c}}$ & $2.0(1.1)$ & $2.0(1.1)$ & $1.6(0.9)$ & $1.7(1.1)$ & $1.5(1.1)$ & $1.4(0.8)$ \\
\hline \multicolumn{7}{|l|}{ Mean blood pressure, mm Hg (SD) } \\
\hline Systolic ${ }^{\mathrm{c}}$ & $139(18)$ & $137(18)$ & $133(18)$ & $135(18)$ & $131(18)$ & $129(19)$ \\
\hline Diastolic $^{c}$ & $85(11)$ & $84(11)$ & $81(11)$ & $82(11)$ & $79(11)$ & $77(11)$ \\
\hline Mean $\mathrm{HbA}_{1 \mathrm{c}}, \%(\mathrm{SD})^{\mathrm{c}}$ & $5.4(0.7)$ & $5.3(0.8)$ & $5.2(0.6)$ & $5.2(0.6)$ & $5.1(0.6)$ & $5.1(0.5)$ \\
\hline Mean BMI $\left(\mathrm{kg} / \mathrm{m}^{2}\right)(\mathrm{SD})^{\mathrm{c}}$ & $28.8(3.4)$ & $27.5(3.6)$ & $24.5(3.4)$ & $26.1(3.8)$ & $24.2(3.2)$ & $22.9(1.5)$ \\
\hline Mean fat intake, g/day $(\mathrm{SD})^{\mathrm{c}}$ & $85.1(29.1)$ & $85.2(28.9)$ & $78.4(28.5)$ & $54.2(18.0)$ & $48.1(16.2)$ & $42.2(14.6)$ \\
\hline Mean saturated fat intake, g/day $(\mathrm{SD})^{\mathrm{c}}$ & $33.7(12.7)$ & $33.5(12.7)$ & $30.5(12.5)$ & $19.0(6.7)$ & $16.1(5.5)$ & $13.3(5.0)$ \\
\hline Mean fibre intake, $\mathrm{g} /$ day $(\mathrm{SD})^{\mathrm{c}}$ & $16.8(6.0)$ & $17.7(6.0)$ & $18.3(6.1)$ & $20.0(6.9)$ & $22.2(8.3)$ & $29.0(8.3)$ \\
\hline Mean number of hours of physical activity/week (SD) ${ }^{c}$ & $1.7(1.2)$ & $10.4(11.0)$ & $11.3(10.5)$ & $11.2(10.7)$ & $12.3(10.8)$ & $12.0(9.2)$ \\
\hline
\end{tabular}

${ }^{a}$ Numbers for each variable might not add up to total because of missing values

${ }^{\mathrm{b}} \chi^{2}$ test for trend, $p<0.001$

cANOVA, $p<0.001$

${ }^{\mathrm{d}} \chi^{2}$ test comparing current with ex/never smokers, $p<0.001$

Kruskal-Wallis test, $p<0.001$ 


\section{Results}

Baseline characteristics for the study population are shown in Table 1. Those meeting a higher number of goals were more likely to be female, younger, from a higher social class and to have never smoked than those meeting fewer goals ( $\chi^{2}$ test for trend, $\left.p<0.001\right)$. Similar trends were also evident for other associated risk factors: participants who met fewer goals were more likely to have larger waist and hip circumferences, higher lipid values (total cholesterol, LDL and triglycerides), higher $\mathrm{HbA}_{1 \mathrm{c}}$ and elevated blood pressure readings, as well as lower HDL values ( $p$ values were $<0.001$ for all continuous variables). Those meeting more goals were also more likely to have a lower BMI, to consume less fat and saturated fat, to consume more fibre, and to engage in more physical activity. Apart from the BMI and fibre goals, the proportion of participants meeting each diabetes prevention behaviour goal was similar to figures reported for the control group in the DPS [2]. Only one-fifth of EPIC participants met three or more diabetes prevention behaviour goals, $10 \%$ achieved no goals, and just $1 \%$ achieved all five goals.

There were 394 incident cases of diabetes during a mean follow-up of 4.6 years (range 2-7 years, total person years of follow-up, 110,079). The cumulative incidence rate of diabetes was 3.5 per 1,000 person-years. The number of participants achieving each of the diabetes prevention behaviour goals is shown in Table 2. Only $3.8 \%$ of participants reported consuming at least $15 \mathrm{~g} / 4,184 \mathrm{~kJ}$ of fibre daily, but $76.0 \%$ reported engaging in physical activity for more than $4 \mathrm{~h}$ a week. The incidence of diabetes was inversely related to the number of diabetes prevention goals achieved ( $\chi^{2}$ test for trend, $p<0.001$ ) (Fig. 1). No participant who met all five goals developed diabetes; the incidence of diabetes was highest in those who did not meet any goals. If the entire population was able to meet one more goal, then the total incidence of diabetes would be predicted to fall by $20 \%$. The Cox regression analysis demonstrated that the hazard ratios for diabetes prevention scores were not significantly attenuated after adjustment for age, sex, social class, family history of diabetes and prescription of antihypertensive medication. After recoding BMI, fibre intake and exercise into quintiles, we found a strong linear trend with incident diabetes. The single diabetes prevention goal which appeared to confer the most protection against developing diabetes was a BMI $<25 \mathrm{~kg} / \mathrm{m}^{2}$, with an adjusted odds ratio of $0.21(95 \% \mathrm{CI}$ 0.15-0.29), and this was followed by exercising at least $4 \mathrm{~h}$ a week (odds ratio $0.76 ; 95 \%$ CI $0.61-0.95$ ). Saturated fat intake was significantly associated with the development of diabetes, but fat and fibre intakes were not (Table 2). In terms of weight difference in the baseline date, both the crude and the adjusted relative risk was 0.78 for a reduction of $1.5 \mathrm{~kg} / \mathrm{m}^{2}$ (BMI) units.

Sensitivity analyses demonstrated that missing healthy goal exposure data did not affect our model: the linear trend remained statistically significant when recoding to maximum or minimum values $\left(\chi^{2}\right.$ test for trend, $\left.p<0.001\right)$. Stratifying the model by sex demonstrated that a larger proportion of females met each of the DPS healthy behaviour goals (except for exercise $>4 \mathrm{~h} /$ week), and that the incident rate of diabetes was over 1.5 times higher for men than for women $(p<0.001)$. The general trend remained the same for both sexes $\left(\chi^{2}\right.$ test for trend, $p<0.001$, data not shown).

\section{Discussion}

Few participants in this population-based observational cohort study were meeting diabetes healthy behaviour goals. The distribution was similar to figures reported for the control group with IGT in the DPS. Similarly, Reeves and Rafferty have confirmed that only $3.0 \%$ of Americans meet all of a set of four comparable healthy lifestyle characteristics [13]. We demonstrated that people who met more of a set of five health behaviour goals for diabetes prevention were less likely to develop the condition in the future. The incidence of diabetes was inversely and linearly related to the number of goals achieved. It is clear that participants who achieved more of the diabetes healthy behaviour goals differed in important ways from those who achieved fewer goals. Those meeting higher numbers of goals were more likely to be female, younger, from a higher social class and to have never smoked than those meeting fewer goals. Participants who met fewer goals were also more likely to have a number of related risk factors, including larger waist circumference, higher total cholesterol and elevated blood pressure. They also had higher BMI, consumed more fat and saturated fat, consumed less

Table 2 Numbers of respondents achieving each diabetes healthy behaviour goal at baseline, and association of each goal with incident diabetes: the EPIC-Norfolk study 1993-2000 $(n=24,155)$

\begin{tabular}{lcccc}
\hline & Number & Percentage & Univariate odds ratio $^{\text {Multivariate odds ratio }^{\mathrm{a}}}$ \\
\hline $\mathrm{BMI}<25 \mathrm{~kg} / \mathrm{m}^{2}$ & 9,632 & 39.9 & $0.18(0.13-0.25)^{\mathrm{b}}$ & $0.21(0.15-0.29)^{\mathrm{b}}$ \\
Fat intake $<30 \%$ of energy intake & 6,646 & 27.5 & $1.06(0.85-1.32)$ & $1.28(0.98-1.68)$ \\
Saturated fat intake $<10 \%$ of energy intake & 4,642 & 19.2 & $0.80(0.61-1.05)$ & $0.71(0.51-0.99)^{\mathrm{c}}$ \\
Fibre intake $\geq 15 \mathrm{~g} / 4184 \mathrm{~kJ}$ & 912 & 3.8 & $0.73(0.40-1.33)$ & $0.83(0.44-1.56)$ \\
Exercise $>4 \mathrm{~h} /$ week & 18,352 & 76.0 & $0.71(0.57-0.88)^{\mathrm{c}}$ & $0.76(0.61-0.95)^{\mathrm{c}}$ \\
\hline
\end{tabular}

\footnotetext{
${ }^{a}$ Adjusted for all other diabetes behaviour scores, age, sex, social class, family history of diabetes and prescription of antihypertensive medication

${ }^{\mathrm{b}} p<0.001$

${ }^{c} p=0.001-0.05$
} 


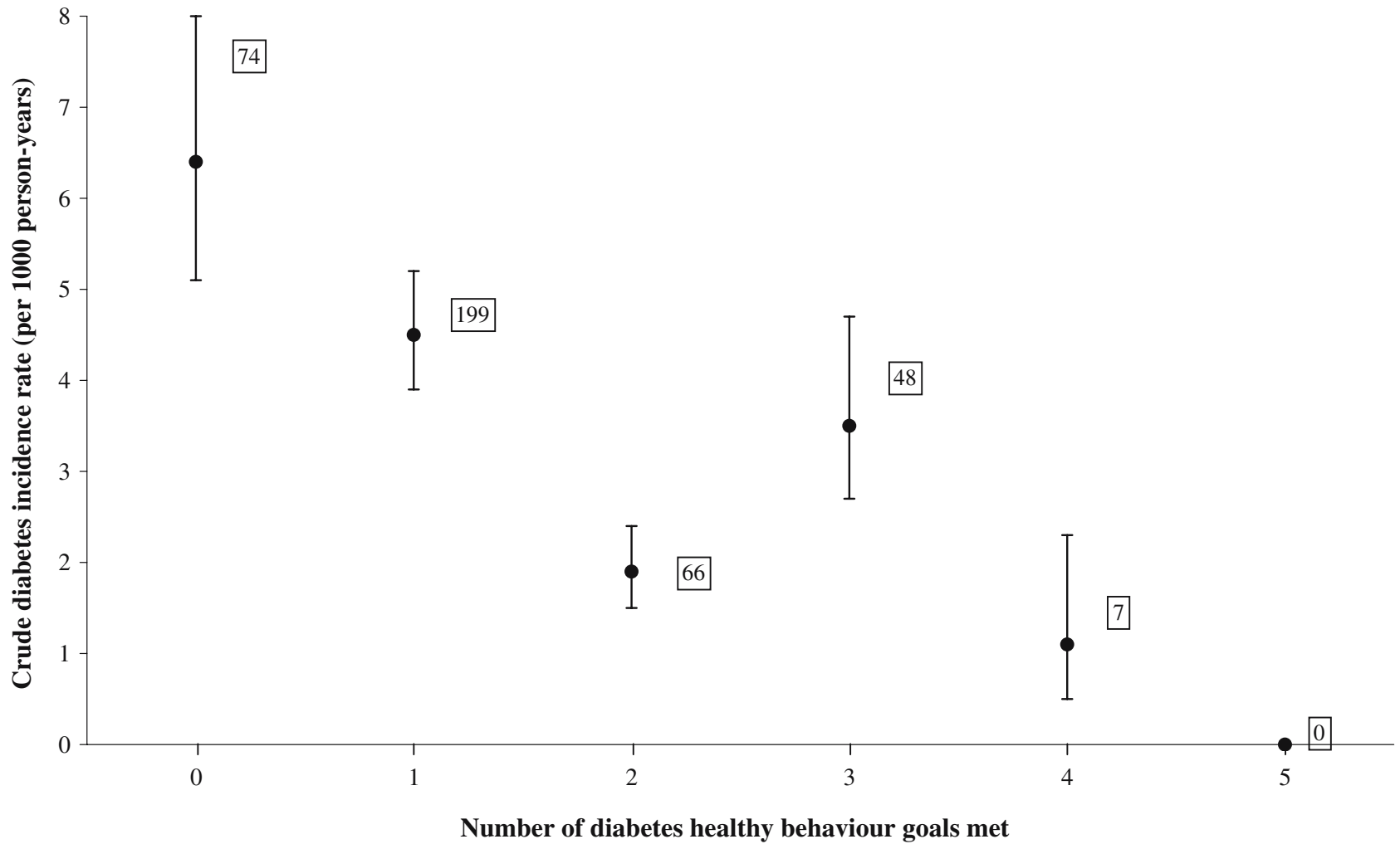

Fig. 1 Crude incidence of diabetes according to the number of diabetes healthy behaviour goals achieved: the EPIC-Norfolk study 1993$2000(n=24,155)$. Vertical lines refer to $95 \%$ CIs; boxes refer to the number of incident diabetes cases for each number of goals met

fibre, and engaged in less physical activity. Evidence of colinearity between the behaviour goals demonstrated that unhealthy behaviours tended to cluster. This finding has implications for the types of people who should be targeted by health promotion interventions to prevent progression to diabetes. However, even after adjustment for age, sex, social class, family history of diabetes and the prescription of antihypertensive medication, results from the Cox regression analysis suggest that the diabetes behaviour goals are important in predicting incident diabetes and therefore provide goals that might be targeted in preventive strategies.

EPIC-Norfolk is a large population-based cohort and high ascertainment of diabetes incidence was achieved [12]. The overall incidence of diabetes was similar to figures reported in other UK studies looking at comparable populations [14]. Some selection bias may be present as people attending general practice surgeries who were not fulfilling the behavioural goals may have been more likely to be tested, and consequently diagnosed with diabetes (for example, if they were visibly overweight), or reported an unhealthy diet and/or low levels of physical activity. However, as we achieved $99 \%$ case ascertainment for all EPIC-Norfolk participants, whether or not they returned for the second health check, few clinically incident diabetic individuals are likely to have been missed. Furthermore, incident diabetes was also defined biochemically using $\mathrm{HbA}_{1 \mathrm{c}}>7 \%$, so the effect of selection bias is unlikely to be large.
The original behaviour goal related to obesity in the Finnish DPS was to achieve a reduction of at least $5 \%$ in body weight to reach a target BMI of less than $25 \mathrm{~kg} / \mathrm{m}^{2}$. As we used single baseline data from EPIC in this prospective cohort analysis, we did not include change in weight data, but simply stratified the population by whether or not participants had a BMI above or below the target value of $25 \mathrm{~kg} / \mathrm{m}^{2}$. The effect of dichotomising the weight target would be to underestimate the benefits of weight reduction in seriously obese individuals. For example, a $5 \%$ weight reduction in a six-foot $(1.83 \mathrm{~m})$ man initially weighing $120 \mathrm{~kg}$ would reduce the BMI from 35.4 to $33.7 \mathrm{~kg} / \mathrm{m}^{2}$. Although such a weight reduction is likely to give rise to health benefits, it would not allow such an individual to meet the behaviour goal as we have defined it. Indeed, only by losing $36 \mathrm{~kg}$ or $30 \%$ of body weight, which is almost impossible, would such an individual attain the BMI target. However, by calculating the relative risk reduction for each $1.5 \mathrm{~kg} / \mathrm{m}^{2}$ difference in BMI, which is roughly equivalent to a $5 \%$ reduction in weight for most people, we demonstrated a risk reduction of around $20 \%$. Thus, it may be by expressing the target in terms of a greater number of levels rather than simply dichotomising it at $25 \mathrm{~kg} / \mathrm{m}^{2}$ that more realistic targets can be developed. When we recoded BMI into quintiles, there was a strong inverse linear trend with incident diabetes. Similar trends were also seen across the fibre and exercise DPS healthy behaviour goals after recoding them into quintiles. This suggests that even if the goals are not met, relatively small shifts of the entire population towards more healthy 
behaviour would result in a reduction in the incidence of diabetes.

In terms of the physical activity DPS goal, we created a similar composite variable that included self-reported hours per week of walking, cycling and 'other physical activity'. The number of participants meeting this goal (76\%) approximated values from the Finnish control data. Results for the number of people meeting fat and saturated fat goals were roughly similar to those found in the DPS. We demonstrated that crude saturated fat intake was not significantly associated with the development of diabetes, but in multivariate analysis it showed a slight protective effect. As the other dietary behaviours were simultaneously adjusted for in this model, it is difficult to elucidate the contribution of specific dietary factors in diabetes progression - the finding perhaps indicates low overall fat intake and possibly high intake of refined carbohydrate. The fibre goal was achieved by just $4 \%$ of the EPIC population. EPIC uses the Englyst and Cummings non-starch polysaccharide definition of fibre [15]; this tends to give slightly lower values than the Southgate method [16], which includes other types of fibre in addition to non-starch polysaccharide. However, verification of daily fibre intake from other EPIC populations around Europe showed that the UK values were particularly low [17]. This suggests that the DPS fibre goal is unrealistic for the majority of people on a British diet and much work needs to be done to improve national dietary patterns.

We have shown that achievement of diabetes healthy behaviour goals is associated with incident diabetes in a large population-based cohort. This has implications for individual- and population-level preventive strategies. Information from this analysis could be used to identify those at high risk of developing diabetes. Conducting oral glucose tolerance tests may not be a feasible population strategy for finding people at high risk. By collecting simple information about the health behaviours identified in the DPS it may be possible to target a group that is more likely to go on to develop diabetes. Our analyses also demonstrated that unhealthy behaviours tend to cluster in particular subgroups: deprived older males with a history of smoking were at increased risk of developing diabetes. Although it is clear which subgroups are at increased risk, it remains unclear whether targeting people in adverse circumstances with adverse health behaviours will lead to significant public health gain. Consequently, there is also a need to identify those who are most amenable to change. Finally, we must develop effective interventions that target larger groups of people at lower absolute risk. The sort of intensive behavioural interventions evaluated in the diabetes prevention trials are costly and time-consuming and, although they are cost-effective in high risk individuals, may not be either feasible or cost-effective for the very much larger group of people at lower absolute risk. Results from these analyses suggest that population-level interventions should target increases in physical activity alongside decreases in BMI, and point to small achievable goals concerning nutritional intake, physical activity and weight rather than the achievement of all-or-nothing dichotomous goals. Thus, interventions that promote movement towards the healthy behaviour goals for diabetes prevention, if successfully applied in the general population, could significantly reduce the growing burden of diabetes-related morbidity and mortality. As $80 \%$ of EPIC participants managed to meet only two of the diabetes prevention goals, there is considerable scope for movement across these behaviours. Future research should therefore focus on developing low-cost interventions that target larger groups of people to tackle the rising incidence of diabetes.

Acknowledgements We gratefully acknowledge the contributions of EPIC-Norfolk participants, the EPIC-Norfolk team, particularly J. Camus and R. Luben, and the different funding bodies: British Heart Foundation; Cancer Research UK; Department of Health; Food Standards Agency; European Union (Europe Against Cancer Programme); Ministry of Agriculture, Fisheries and Food; Medical Research Council; Stroke Association; Wellcome Trust; and the World Health Organization.

\section{References}

1. Pan XR, Li GW, Hu YH et al. (1997) Effects of diet and exercise in preventing NIDDM in people with impaired glucose tolerance. The Da Qing IGT and Diabetes Study. Diabetes Care 20:537-544

2. Tuomilehto J, Lindstrom J, Eriksson JG et al. (2001) Prevention of type 2 diabetes mellitus by changes in lifestyle among subjects with impaired glucose tolerance. $\mathrm{N}$ Engl J Med 344:1343-1350

3. Knowler WC, Barrett-Connor E, Fowler SE et al. (2002) Reduction in the incidence of type 2 diabetes with lifestyle intervention or metformin. N Engl J Med 346:393-403

4. Day N, Oakes S, Luben R et al. (1999) EPIC-Norfolk: study design and characteristics of the cohort. European Prospective Investigation of Cancer. Br J Cancer 80(Suppl 1):95-103

5. McKeown NM, Day NE, Welch AA et al. (2001) Use of biological markers to validate self-reported dietary intake in a random sample of the European Prospective Investigation into Cancer United Kingdom Norfolk cohort. Am J Clin Nutr 74:188-196

6. Wareham NJ, Jakes RW, Rennie KL et al. (2003) Validity and repeatability of a simple index derived from the short physical activity questionnaire used in the European Prospective Investigation into Cancer and Nutrition (EPIC) study. Public Health Nutr 6:407-413

7. Jakes RW, Day NE, Luben R et al. (2004) Adjusting for energy intake - what measure to use in nutritional epidemiological studies? Int J Epidemiol 33:1382-1386

8. Office for National Statistics (2004) Deaths by health area of usual residence, numbers and standardised mortality ratios (SMRs) by sex, 2004 registrations. Available from http://www.statistics.gov.uk/STATBASE/Expodata/Spread sheets/D9076.xls, last accessed 25 January 2006

9. Welch AA, Luben R, Khaw KT, Bingham SA (2005) The CAFE computer program for nutritional analysis of the EPICNorfolk food frequency questionnaire and identification of extreme nutrient values. J Hum Nutr Diet 18:99-116

10. Rowley KG, Daniel M, O'Dea K (2005) Screening for diabetes in Indigenous populations using glycated haemoglobin: sensitivity, specificity, post-test likelihood and risk of disease. Diabet Med 22:833-839

11. McCance DR, Hanson RL, Charles MA et al. (1994) Comparison of tests for glycated haemoglobin and fasting and two hour plasma glucose concentrations as diagnostic methods for diabetes. BMJ 308:1323-1328 
12. Harding AH, Day NE, Khaw KT et al. (2004) Dietary fat and the risk of clinical type 2 diabetes: the European prospective investigation of Cancer-Norfolk study. Am J Epidemiol 159:73-82

13. Reeves MJ, Rafferty AP (2005) Healthy lifestyle characteristics among adults in the United States, 2000. Arch Intern Med 165:854-857

14. Perry IJ, Wannamethee SG, Walker MK, Thomson AG, Whincup PH, Shaper AG (1995) Prospective study of risk factors for development of non-insulin dependent diabetes in middle aged British men. BMJ 310:560-564
15. Englyst H, Wiggins HS, Cummings JH (1982) Determination of the non-starch polysaccharides in plant foods by gas-liquid chromatography of constituent sugars as alditol acetates. Analyst 107:307-318

16. Southgate DA (1969) Determination of carbohydrates in foods II. Unavailable carbohydrates. J Sci Food Agric 20:331-335

17. Bingham SA, Day NE, Luben R et al. (2003) Dietary fibre in food and protection against colorectal cancer in the European Prospective Investigation into Cancer and Nutrition (EPIC): an observational study. Lancet 361:1496-1501 